\title{
Controllo della performance nelle aziende dei servizi: contributi e tendenze
}

\author{
Selena Aureli, Claudio Travaglini*
}

\begin{abstract}
Performance evaluation in service companies. Contributions and trends

This issue of Management Control focuses on the so-called service sector or tertiary sector, which represents the most important component of every country's economy. Since this sector covers a wide variety of activities, this issue gathers contributions on performance measurement systems and metrics used in different types of service businesses, such as transport companies, restaurants, public utilities, financial institutions, software companies and organizations delivering healthcare services.

Reasons for a thematic issue on performance evaluation in service companies are: the speed at which modern countries have made the transition to service-based economies, the emergence of new Internet-based services and ways of using services due to the spread of ICTs and the demand for new management control systems or metrics capable to respect services' characteristics and their trends of change like the increasing involvement of costumers in service production.

Selected contributions coherently highlight the need to integrate financial performance metrics with the measurement of qualitative and non-monetary results like customer satisfaction and socio-environmental impacts, the shift from monitoring processes to focusing on service outcomes and the opportunity offered by ICTs to collect and elaborate a great amount of data on customers, processes and external sources and use them to improve service managers' decision making.
\end{abstract}

Keywords: management control, ICT, public services, performance measurement, service co-production.

\section{Introduzione}

La presente special issue raccoglie alcuni contributi selezionati presentati al $\mathrm{V}$ Management Control Journal Workshop, dedicato ai sistemi di controllo nelle aziende di servizi. La scelta del tema o meglio del settore in cui trovano applicazione

* Dipartimento di Economia Aziendale, Università di Bologna - Campus di Rimini, $\underline{\text { se- }}$ lena.aureli@unibo.it; claudio.travaglini@unibo.it. 
tanti e diversi strumenti di controllo, in origine tipicamente sviluppati nell'ambito dell'industria manifatturiera, è riconducibile a tre ordini di motivi:

- la crescente rilevanza del settore dei servizi a livello mondiale;

- l'emergere di nuovi servizi e di nuove modalità di fruizione dei servizi a seguito della diffusione delle ICT;

- la rinnovata istanza proveniente dal mondo accademico di sviluppare specifici sistemi ed approcci di studio al controllo di gestione dei servizi che ne considerino le peculiari caratteristiche ed alcuni loro trend di cambiamento.

Per quanto riguarda il primo punto, è innegabile che il settore dei servizi rappresenti una componente importante dell'economia di qualsiasi nazione. In 138 Paesi, esso contribuisce a più del $50 \%$ del prodotto interno lordo (World Economic Forum, 2009) e concorre direttamente alla creazione di posti di lavoro (Anxo e Storrie, 2001). Inoltre, i servizi sono fondamentali per lo sviluppo di tutte le altre attività produttive, come nel caso delle telecomunicazioni, del trasporto e dei servizi finanziari, e per la crescita sociale ed il benessere della popolazione. Sanità, istruzione e servizi sociali rendono possibile la costruzione ed il mantenimento del "capitale umano", elemento determinante dello sviluppo economico e sociale assieme alla disponibilità di risorse naturali. Studiare strategie e sistemi manageriali con cui le aziende di servizi perseguono i propri obiettivi di sviluppo e contengono i costi, significa dunque riflettere su come migliorare efficacia ed efficienza di quelle organizzazioni che costituiscono uno dei principali drivers di sviluppo dell'Europa e che ci consentono lo stile di vita che identifichiamo come quello di un paese moderno.

Il terziario è il settore che più ha beneficiato della pervasiva e continua diffusione delle tecnologie informatiche, le quali hanno aperto e costruiscono continuamente nuove opportunità di business. Le ICT non solo hanno favorito la nascita di nuovi servizi Internet-based, con una rilevante innovazione di prodotto-servizio, ma hanno anche cambiato i processi aziendali interni ed il modo di interagire con i clienti con una continua azione di innovazione di processo. Soprattutto nel turismo e nel settore bancario, le ICT hanno automatizzato molti processi amministrativi, eliminato manodopera diretta non specializzata, sostituito processi standardizzati con modalità di lavoro a progetto (Holtgrewe, 2015) e modificato la struttura dei costi e la loro rendicontazione (Hyvönen et al., 2009), come quando si ricorre, ad esempio, al cloud computing (Caldarelli et al., 2016). La tecnologia mobile è la dimostrazione di come le ICT possano cambiare il modo di acquistare servizi, senza più doversi recare presso il negozio, la banca o persino il medico, aprendo altresì la possibilità di raccogliere una miriade di dati sui clienti, che le aziende integrano nei sistemi di controllo e di business intelligence (Gatti, 2011). In certi casi le ICT cambiamo anche il modo di produrre i servizi, dando luogo al fenomeno della co-produzione del servizio da parte del cliente (Meijeer, 2012; Palumbo, 2015).

Da ultimo, è utile ricordare come la recente letteratura sulla servitization (Neely, 2008) e la service-dominant logic (Vargo e Lusch, 2004) abbia riaperto la discussione mai conclusa e forse superata sulla distinzione prodotto versus servizio e, conseguentemente, sulla presenza di elementi peculiari nei processi di erogazione dei servizi che fanno sorgere problemi manageriali differenti, a cui occorre dare risposta 
con strumenti e sistemi di performance measurement idonei (Tenucci et al., 2013). Le caratteristiche distintive dei servizi, tradizionalmente identificate nella intangibilità, deperibilità, eterogeneità e inseparabilità tra produzione e consumo, sono infatti alla base del motivo per cui alcuni autori suggeriscono di superare la distinzione tra costi di prodotto e costi di periodo (Auzar e Langfield-Smith, 2005; Modell, 1996), effettuare una diversa analisi della formazione del valore e del costo di produzione (Travaglini, 1996) ed introdurre ulteriori indicatori di performance qualitativi grazie ai quali verificare la soddisfazione del cliente. In particolare, l'inseparabilità tra produzione e consumo fa venir meno il principio della controllabilità del risultato finale (le aspettative e le azioni del cliente introducono un'elevata variabilità sugli outcomes) e quindi rende difficile la valutazione tradizionale delle performance. Per tale motivo, diventa più importante conoscere il "cost of use" (da confrontare con il valore di uso) piuttosto che il "cost of production", ricorrendo all'ausilio di strumenti di costing più idonei come, ad esempio, il total cost of ownership (Ellram, 1993).

In tale scenario, i contributi qui selezionati approfondiscono alcune importanti tendenze evolutive, ancorché non esaustive, che caratterizzano il settore terziario:

○ la mutata soggettività nell'erogazione e nel controllo dei servizi pubblici con il passaggio dal controllo di processo a quello di risultato;

- l'integrazione della sostenibilità sociale ed ambientale nel controllo delle performance dei servizi;

- la crescente partecipazione dei clienti al processo di erogazione dei servizi;

- la possibilità per le aziende di disporre, grazie alle ICT, di una grande quantità di dati sui processi produttivi interni, sui clienti che compartecipano alla produzione dei servizi e su variabili esterne;

- la presenza del rischio e della sua gestione.

\section{Alcuni trend sull'evoluzione delle aziende di servizi e della valuta- zione della loro performance}

Un primo importante cambiamento che in questi ultimi anni ha mutato il mondo dei servizi ed il modo con cui valutarne la performance complessiva, esprimibile nelle sue componenti economico-reddituale, sociale, ambientale e relazionale, riguarda la gestione dei servizi di pubblico interesse (sanità, scuola, trasporto, ecc.). In questo ambito specifico, la ricerca di una maggiore efficienza ha provocato l'emergere di modelli di convenzionamento ed accreditamento o di affidamento in gestione che richiedono una riflessione sia sul concetto di servizio pubblico che sull'enfasi del controllo di gestione.

Ad esempio, nei servizi alla persona, oggetto del contributo di Cattaneo e Bassani, abbiamo non solo soggetti a capitale pubblico, costituiti in forma istituzionale privata, che svolgono servizi pubblici secondo una logica privatistica, ma anche soggetti privati che erogano servizi pubblici con finanziamento pubblicistico e in forza 
di convenzioni che ne regolano l'erogazione. Dunque, il concetto di servizio pubblico cambia da 'servizio prodotto ed erogato dal soggetto pubblicistico' a 'servizio offerto con finanziamento pubblico da parte di un soggetto erogatore di tipo pubblico o privato'. Poiché quest'ultimo deve riuscire a creare valore pubblico (es. il miglioramento della salute dei cittadini), facendo attenzione a coprire i costi realmente sostenuti con le tariffe stabilite dall'ente pubblico, diventa necessario dotarsi di strumenti di misurazione più sofisticati, grazie ai quali valutare l'uso delle risorse e controllare sia i processi produttivi che i risultati conseguiti.

La complessità di gestione della performance si esprime al massimo livello nei servizi sanitari, poiché in questo campo, la graduale ma continua migrazione dal riconoscimento dei costi storici ai sistemi tariffari costringe gli operatori pubblici e privati ad una continua azione di ricerca dell'economicità, anche in relazione al perseguimento di risultato in termini di corretto esito. Questo richiede ai dirigenti sanitari l'attuazione di un sistematico confronto tra valore riconosciuto ai servizi in sede di tariffazione e costi dei fattori produttivi interni che rappresenta la principale determinante dell'economicità dei servizi sanitari (a cui fa riferimento il contributo di Cattaneo e Bassani).

Considerazioni similari valgono anche per il settore della gestione dei rifiuti urbani, su cui si concentra il contributo di Bartolacci, Paolini, Quaranta e Soverchia. Le aziende di igiene urbana esaminate dagli autori devono infatti erogare un servizio di interesse pubblico preservando l'efficienza e la redditività, a cui si aggiunge l'obiettivo di minimizzare l'impatto ambientale complessivo.

Il coniugare gli aspetti sociali ed ambientali con il perseguimento di risultati economico-reddituali soddisfacenti, rappresenta una modalità di azione con cui tutte le aziende devono confrontarsi per conferire adeguata sostenibilità al proprio operare, mantenere buone relazioni con gli stakeholders e così perseguire uno sviluppo di medio-lungo periodo. Nelle organizzazioni non-profit e nelle imprese sociali, gli obiettivi socio-ambientali rappresentano la ragione stessa della costituzione dell'azienda, mentre l'obiettivo economico è un semplice vincolo da rispettare per mantenere la continuità aziendale. In questi casi (come quello analizzato nel contributo di Baldarelli), la valutazione dell'economicità richiede metodologie specifiche, come il Social Return on Investment (SROI), capaci di sintetizzare sia l'impatto economico che quello sociale. Nel caso dei servizi di interesse pubblico, invece, è soprattutto la natura del servizio trattato, che amplia l'accountability di queste aziende verso i portatori di interesse alla dimensione socio-ambientale. Per le aziende di igiene urbana, quindi, diventa fondamentale misurare come i comportamenti ambientali virtuosi (una migliore performance non finanziaria espressa in termini di maggiore raccolta differenziata) possano influire sulle performance economiche.

Il caso analizzato da Bartolacci et al. è utile anche per evidenziare quanto la partecipazione dei clienti-cittadini alla produzione del servizio assuma un ruolo fondamentale nella determinazione dei risultati aziendali. La promozione e condivisione di comportamenti virtuosi (la raccolta differenziata), che qualificano il servizio, non 
sono più considerati come attinenti alla sfera privata dei cittadini. Essi vengono monitorati perché rappresentano elementi della performance ambientale e sono determinanti della performance economico-finanziaria del servizio.

Considerando il processo di co-produzione del servizio da parte del cliente in modo più ampio, dobbiamo prendere atto di come questo protagonismo da parte dei fruitori dei servizi abbia generato un'altra interessante tendenza: la sempre maggior disponibilità di una grande quantità di dati sui bisogni, preferenze di consumo, attitudini e driver di soddisfazione dei clienti. Durante l'interazione con l'azienda erogatrice, il cliente lascia moltissime "briciole tecnologiche" che permettono di fare profilazione del cliente e capire quali servizi o modelli di business siano vincenti. Il trattamento sistematico di grandi quantità di informazioni (i big data), sia di origine interna che esterna, permette di valutare il livello di qualità dei servizi erogati e fare attività di benchmarking, come nel caso delle aziende aeroportuali analizzate nel contributo di Fasone e Puglisi. Inoltre, le ICT permettono di elaborare queste informazioni in modo innovativo, sì da generare indicazioni utili ai processi decisionali del management ed anche al controllo strategico, come dimostra il contributo di Visani nell'analizzare un'azienda distributrice di software. Soprattutto nel mondo dei servizi, dove la soddisfazione del cliente ed altre variabili qualitative giocano un ruolo fondamentale nel determinare il successo aziendale, le ICT permettono di meglio comprendere quali sono i veri fattori critici di successo e, conseguentemente, forniscono un valido aiuto nel definire degli indicatori sintetici di performance più idonei, non limitati al mero calcolo della redditività per l'azionista.

L'aumentata complessità che devono affrontare le aziende di servizi a seguito dei trend sopra evidenziati, si deve poi confrontare con la dimensione rischio (indagata nel paper di Rizzotti e Frisenna in riferimento al rischio di inadempienza nelle banche), che richiede lo sviluppo di un approccio integrato al risk management. Nelle banche e nelle aziende assicurative la gestione del rischio finanziario è centrale, ma essa va affiancata ed integrata con la gestione di altre tipologie di rischio che possono minare la continuità della prestazione. Oggi, queste ed altre aziende di servizi devono tenere conto dei possibili rischi tecnologici (es. perdita dei dati, furto di informazioni dovuto ad hacker, interruzione di servizio dovuto a malfunzionamento dei computer), di quelli socio-ambientali, reputazionali, di tipo macro-economico, di non adeguatezza del servizio prestato (che deve essere sostituito o ripetuto) e di natura legale o regolamentare. Questi ultimi sono rilevanti soprattutto quando ci riferiamo alle aziende che erogano servizi di interesse pubblico, come il trasporto, la distribuzione di energia o la gestione dei rifiuti, in cui l'ente pubblico, da un lato, e il cliente, dall'altro, possono modificare lo scenario di riferimento rispettivamente con l'emanazione di nuove leggi o mediante pressioni socio-politiche (Aureli e Salvatori, 2013). 


\section{Il cambiamento del modello di controllo nei processi di erogazione dei servizi: dal controllo di processo al controllo di esito}

Rispetto alle diverse tendenze evolutive che possiamo riconoscere all'interno del mondo dei servizi, appare particolarmente rilevante, a parere di chi scrive, focalizzare l'attenzione sul passaggio dal controllo di processo a quello di risultato o esito del servizio nelle aziende erogatrici di servizi pubblici (educativi, sanitari, sociali, ecc.). In questo ambito specifico, infatti, i mutamenti legislativi e di contesto che hanno accompagnato l'avvento del New Public Management (Hood, 1995; Hood e Peters, 2004; Anessi-Pessina e Steccolini, 2005), hanno generato importanti cambiamenti in termini di controllo di gestione e misure di performance da monitorare.

In passato, a fronte di un modello unico di produzione ed erogazione del servizio basato su regole e programmazione uniforme, il controllo si concentrava sulle modalità di produzione e quindi sulla successione delle operazioni di gestione, presumendo che la corretta effettuazione delle operazioni di gestione determinasse una parallelamente corretta erogazione. In questo quadro il controllo è un controllo procedurale e la revisione legale dei requisiti, insieme alla revisione gestionale di procedure basata sulla verifica degli standard organizzativi e di erogazione, è il paradigma tipico del controllo.

Con l'affidamento (in regime di convenzione o accreditamento) a diversi possibili soggetti della gestione del servizio, l'oggetto di riferimento del controllo deve mutare dal processo al risultato. Quindi, una volta garantiti alcuni requisiti minimi di processo e struttura (es. i medici sono laureati e le attrezzature sono omologate), l'oggetto del controllo della performance non è la corretta esecuzione del processo di erogazione del servizio (se le cure vengono regolarmente erogate), bensì l'esito desiderato (il numero delle complicanze o della mortalità per evento devono essere contenute). Allo stesso modo nelle Università, la performance nella formazione dei laureati viene giudicata complessivamente attraverso gli esiti, ossia attraverso tempi e soddisfazione del loro inserimento nel mondo del lavoro.

Questo passaggio dal controllo di processo al controllo di esito genera un importante effetto: la deregolamentazione del processo che lascia spazio all'innovazione. Se il controllo si concentra sull'esito, è sufficiente un controllo dei requisiti minimi degli elementi. In tal modo si libera la combinazione produttiva e si incentiva l'innovazione, rendendo possibile l'introduzione o l'eliminazione di fasi od elementi del processo di servizio tradizionalmente presenti od assenti, ma giudicati necessari o superflui. Le aziende cercano, cioè, di produrre ed erogare i servizi utilizzando una pluralità di combinazioni produttive, alla ricerca della migliore economicità che gli indicatori di performance tentano di indagare dalle diverse prospettive. 


\section{Bibliografia:}

Anessi-Pessina E. e Steccolini I. (2005), Evolutions and Limits of New Public ManagementInspired Budgeting Practices in Italian Local Governments, Public Budgeting \& Finance, 25(2), pp. 1-14.

Anxo D. e Storrie D. (a cura di) (2001), The job creation potential of the service sector in Europe. Final Report 2000, Employment Observatory Research Network, Luxembourg, European Commission.

Aureli S. e Salvatori F. (2013), Investigation of risk management and risk disclosure practices of Italian listed local utilities, Financial Reporting, Vol. 1.

Auzar S.M., Langfield-Smith K. (2005), The effect of service process type, business strategy and life cycle stage on bureaucratic MCS in service organizations, Management Accounting Research, p. 339-421.

Caldarelli A., Ferri L., Maffei M. (2016), I rischi derivanti dall'implementazione del cloud computing: un'indagine empirica nelle PMI italiane, Management Control, vol. 3, p. 27 48. Doi: 10.3280/MACO2016-003003.

Ellram L.M. (1993), Total cost of ownership: elements and implementation, International Journal of Purchasing and Materials Management, 29(4), p. 3-10.

Doi: 10.1111/j.1745-493X.1993.tb00013.x.

Gatti M. (2011), Gli strumenti di controllo orientate al cliente: un'analisi sistemica, Management Control, vol. 1, p. 99-124. Doi: 10.3280/MACO2011-001005.

Holtgrewe U. (2015), Services research along the service process: An overview study to support UNI Europa's services policy project, Forba (www.forba.at), Vienna.

Hood C. (1995), The "New Public Management" in the 1980s: variations on a theme, Accounting, Organizations and Society, 20, pp. 93-109.

Doi: 10.1016/0361-3682(93)E0001-W.

Hood C. e Peters G. (2004), The Middle Aging Of New Public Management: Into The Age Of Paradox?, Journal Of Public Administration Research And Theory, 14(3), pp. 267282.

Hyvönen T., Järvinen J., Pellinen J., Rahko T. (2009), Institutional Logics, ICT and Stability of Management Accounting, European Accounting Review, 18(2), p. 241-275.

Doi: 10.1080/09638180802681511.

Marchi L. (2014), Nuove prospettive di valutazione delle performance nelle aziende di servizi, Management Control, 1, pp. 5-8. Doi: 10.3280/MACO2014-001001.

Meijer A.J. (2012), Co-production in an Information Age: Individual and Community Engagement Supported by New Media, Voluntas, 23(4), pp. 1156-1172.

Doi: $10.1007 / \mathrm{s} 11266-012-9311-\mathrm{z}$.

Model S. (1996), Management Accounting and Control in Services: structural and behavioural perspectives, International Journal of Service Industry Management, 12(5), p. 451-475. Doi: $10.1108 / 09564239610113479$.

Neely A. (2008), Exploring the financial consequences of the servitization of manufacturing, Operations Management Research, vol. 1, N. 2, p. 103-118.

Doi: 10.1007/s12063-009-0015-5.

Palumbo R. (2015), Una contestualizzazione del concetto di co-produzione al caso dei servizi pubblici: una rassegna critica della letteratura internazionale, Azienda Pubblica, 2, pp.137-161.

Tenucci A., Barontini R., Cinquini L. e Giannetti R. (2013), Models of performance and value measurement in service system", in Cinquini L., Di Minin A. e Varaldo R. (a cura di), 
New Business Models and Value Creation: A Service Science Perspective, Milano, Springer, pp. 105-125.

Travaglini C. (1996), Un metodo innovativo per la determinazione di prezzi e le valutazioni di redditività per i servizi turistici, in L'innovazione nell'economia delle aziende, Napoli, Edizioni Scientifiche Italiane.

Vargo S.P., Lusch R.F. (2008), From goods to service(s): divergences and convergences of logics, Industrial Marketing Management, p. 254-259.

Doi: 10.1016/j.indmarman.2007.07.004.

World Economic Forum (2009), The Global Competitiveness Report 2009-2010, Geneva. 